\title{
ПРИМЕНЕНИЕ АВТОНОМНЫХ СЕНСОРОВ ДЛЯ АВТОМАТИЗАЦИИ НЕФТЕГАЗОДОБЫЧИ
}

\author{
Мамонова Татьяна Егоровна', \\ stepte@tpu.ru
}

\author{
Громаков Евгений Иванович1, \\ gromakov@tpu.ru \\ 1 Национальный исследовательский Томский политехнический университет, \\ Россия, 634050, г. Томск, пр. Ленина, 30.
}

\begin{abstract}
Актуальность. Существенным достоинством автономных сенсоров при использовании их для автоматизации технологических процессов является отсутствие кабельных проводок. Поставщики считают, что их внедрение обеспечивает сокращение стоимости капитальных затрат на строительство объектов (в частности, за счёт экономии на кабельной продукции и материалах), снижение сроков строительно-монтажных и пусконаладочных работ, сокращение энергопотребления и снижение операционных затрат. Перспектива применения проектных решений автоматизации с использованием радио коммуникационной среды передачи данных имеет многообещающий потенциал развития. Внедрение сетецентрических технологий автоматического (автоматизированного) управления на основе интеллектуальных сенсорных сетей, прежде всего, позволит в ближайшей перспективе повысить эффрективность добычи углеводородного сырья на труднодоступных территориях, снизит потребность в обязательном присутствии человека на технологических площадках. Все это, в свою очередь, может значительно изменить проектные решения по автоматизации объектов нефттегазодобычи.

Цель: выявление основных препятствий при использовании автономных сенсоров для автоматизации нефтегазодобычи, проведение сравнительного анализа основных производителей автономных сенсоров.

объекты: сетецентрические технологии автоматического (автоматизированного) управления на основе интеллектуальных сенсорных сетей для нефтегазодобычи.

Методы: системный анализ отечественного и зарубежного рынков автономных сенсоров и сетей.

Результаты. Выполнен анализ применения автономных сенсоров и сетей на их основе. Выявлены основные преимущества применения автоматизации в нефтегазовой отрасли на основе автономных сенсоров. Показаны условия, при которых перспективно применение автономных беспроводных сенсоров на нефртегазовых объектах. В результате выполненного исследования не удалось выявить значимую экономическую эфрфективность автоматизации типовых технологических процессов нефртегазодобычи путем замены всей проводной автоматизации на ее беспроводной аналог. В настоящий момент времени применение автономных сенсорных сетей в области автоматических систем управления технологическими процессами для опасных производственных объектов нефртегазодобьчи также ограничено, поскольку возникают трудности сертиффикации функциональной безопасности. Это, в частности, связано с проблемами отказобезопасности радио коммуникационной среды, а также значительной задержкой технического обслуживания в случае удаленности и труднодоступности технологических объектов управления.
\end{abstract}

\section{Ключевые слова:}

Сенсорные сети, автономные сенсоры, умное месторождение, автоматизированные системы управления, контрольно-измерительные приборы.

\section{Введение}

Как показывают исследования, отражённые в работах [1-9], сенсоры различного назначения используются на объектах нефтегазодобычи, в системах транспортировки и переработки сырья. Практика пилотных проектов позволяет считать, что реализация цифровых и интеллектуальных технологий управления активами нефтегазовой отрасли характеризуется масштабным, быстрым и эффективным возвратом вложенных в них финансовых ресурсов. В крупных компаниях имеются все необходимые условия для технологического прорыва в цифровизации отрасли путем внедрения интеллектуальных систем разработки месторождений, добычи, транспорта и переработки нефти и газа, а также внедрения IOT технологии. С использованием автономных контрольно-измерительных приборов и аппаратуры (КИПиА) перечисленные технологии позволят ускорить реализацию безлюдной эксплуатации нефтегазовых объектов (НГО).

Предполагается, что с распространением автономных сенсоров, работающих от встроенного элемента питания и не требующих кабельных проводок, появ- ляется возможность сократить стоимость капитальных затрат на строительство объектов, снизить сроки строительно-монтажных (СМР) и пусконаладочных работ (ПНР), сократить энергопотребление и снизить операционные затраты. Все это, в свою очередь, может значительно изменить проектные решения по автоматизации объектов нефтегазодобычи.

Целью данной работы является исследование перспектив проектных решений для измерительных систем в области автоматизации (интеллектуализации) технологических процессов нефтегазодобычи на основе автономных сенсоров и самоорганизующихся сенсорных сетей (WSN)

Результат анализа ориентирован на проекты строительства и модернизации производственных объектов крупной нефтегазодобывающей компании, обустройства месторождений нефти и газа, автоматизации (интеллектуализации) технологических процессов нефтегазодобычи. Автономные сенсорные устройства состоят из сенсора, процессора, батареи питания, оптического приемопередатчика или радио приемопередатчика с антенной. Возможны реализации сенсорных блоков с интегральным или раздель- 
ным приёмопередатчиком. В данной работе рассматриваются радио канальные автономные сенсорные устройства и сети на их основе.

Различают следующие направления развития сенсорных сетей.

1. Сенсорные сети типа умная пыль (Smart Dust), решающие задачи измерения, сбора данных на основе радио-канальной или лазерной телекоммуникационной сети микроминиатюрных дешевых датчиков, в ряде случаев разового применения.

2. Сенсорные сети автономных измерительных приборов (WSN), решающие задачи беспроводного сбора данных о состоянии технологического процесса и его оборудования.

3. Интеллектуальные сети КИПиА, решающие задачи сбора данных на основе интеллектуальных измерительных приборов и исполнительных устройств.

Технология Smart Dust только начинает использоваться ведущими нефтегазовыми компаниями и пока находит разовые применения, в частности, при сборе информации о площадях экологического загрязнения, пожаро-распространении и др. Под интеллектуальным измерительным прибором понимается датчик со встроенным вычислительным блоком, который выполняет в реальном времени расчетные задачи, такие как: статистическая обработка данных измерений, вычисление ключевых показателей процессов производств, самовалидация измерений, автокалибровка, самодиагностика и др. Исключительной особенностью интеллектуальных датчиков является их способности самовосстанавливаться и самообучаться после возникшего сбоя (ГОСТ Р 56947-2016/ISO/IEC/IEEE 21450:2010). В зарубежной литературе датчики данного типа именуются «Smart Sensor» (из стандартов IEEE 1451).

Особенным потенциалом развития обладают автономные сенсорные сети, соответствующие требованиям ГОСТ Р ИСО МЭК 29182, сетецентрические IOT-структуры управления производственным процессом на основе NB-Fi протокола национального стандарта ПНСТ 354-2019.

Пример структуры автономной сенсорной сети сбора данных на нефтескважине показан на рис. 1 .

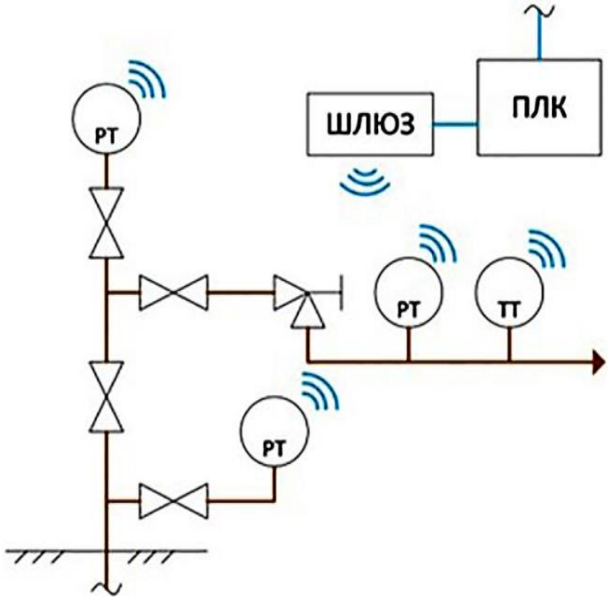

Рисунок. Автономная сенсорная сеть сбора данных на нефтескважине

Figure. Autonomous sensor network of oil well data collection

\section{Системный анализ современных технических решений}

Для сравнительного анализа технических решений, предлагаемых технологическими лидерами в области автоматизации, были выбраны следующие особенности сенсорных устройств, значимые для автоматизации технологических процессов нефтегазодобычи: характеристики полевого исполнения, типы датчиков, готовых для применения на НГО; системные особенности WSN; условия их настройки; безопасность и техническая поддержка. Для сравнительной оценки были использованы технические каталоги продукции, информация из печатных статей российских и зарубежных журналов, отражающие задел мирового научного сообщества и перечисленные в источниках литературы [10-17]. Полученные сведения отечественных компаний «СТРИЖ», «С-sensor», «СИАМ», «Сенсорика» на момент выполнения анализа показали недостаточную готовность датчиков для применения на НГО. Результаты обработки технических данных наиболее готовых автономных сенсоров для применения на НГО для наглядного отображения их ключевых технических характеристик были сведены в таблице.

При рассмотрении вопроса настройки и установки перечисленных автономных сенсоров было определено, что подготовительные работы на местности требуются для большинства из указанных марок, кроме «Emerson Rosemount», «Endress+Hauser» и «Siemens». Программное обеспечение (ПО) и оборудование для настройки контрольно-измерительных приборов многими компаниями предоставляются в комплекте бесплатно.

Был рассмотрен вопрос обеспечения и установки ПО и оборудования для настройки беспроводной сети. Здесь для каждой марки дело обстоит по-разному. В частности, для Emerson Rosemount имеется вебинтерфейс шлюза и бесплатное ПО AMS Wireless Configurator. Компания Endress+Hauser предоставляет также веб-интерфейс шлюза, ПО FieldCare платное как опция. Фирма Honeywell: для работы беспроводной сети требуется приобретение станции управления и точек доступа, требующих внешнее питание и прокладку сетевого кабеля. Компания Yokogawa предлагает 1 лицензию на ПО Field Wireless Management Tool. Schneider Electric имеет ПО Accutech Manager. У Объединения БИНАР ПО поставляется комплектно с разрабатываемыми системами, при этом для осуществления взаимодействия со сторонними системами необходимо наличие базовой станции. Компания Siemens имеет ПО TIA PORTAL/S7.

Указанные характеристики позволяют сделать вывод о высокой степени готовности решений, предлагаемых технологическими лидерами. Технические решения включают в себя не только поставку отдельных устройств (КИПиА, преобразователи), но и предлагают программные решения для мониторинга, анализа и конфигурирования беспроводных устройств, то есть представляют собой законченные решения для применения в АСУ ТП производственных объектов нефтегазодобычи. 


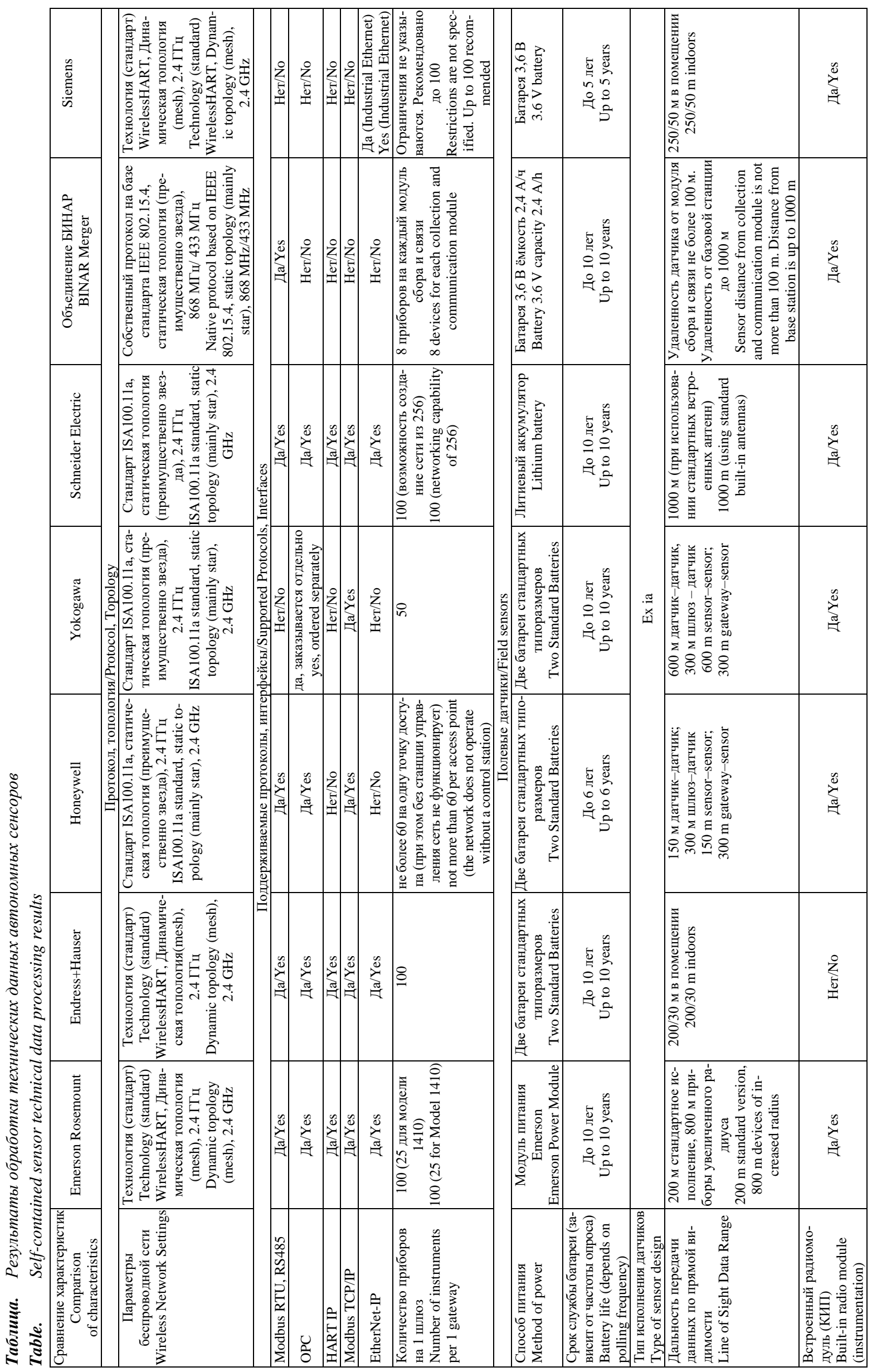




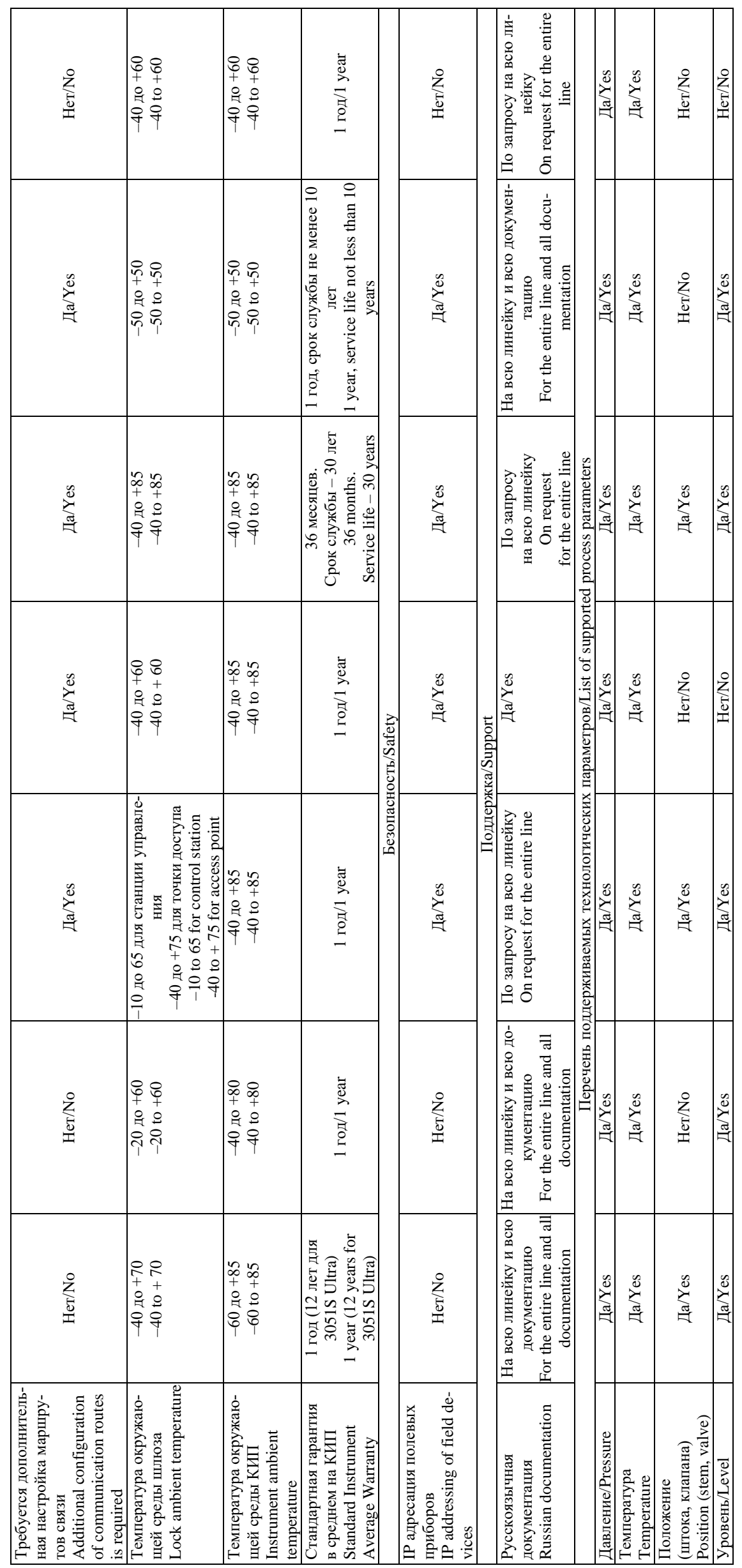


На данный момент основными измеряемыми параметрами с помощью беспроводных сенсоров являются: давление, уровень и температура. Не все производители предлагают датчики расхода, загазованности и т. д. Вызвано это тем, что такие датчики имеют высокое энергопотребление, так как с них постоянно необходимо снимать измерения, что в значительной степени скажется на длительности работы батареи. Использование компаниями Emerson, Rosemount, Endress+Hauser большого количества стандартных сетевых протоколов позволяет обеспечивать альтернативность проектного выбора сетевых средств АСУТП Так, несмотря на существенные достоинства протокола ISA100 (компании Honeywel, Yokogawa, Schneider Electric), известным его недостатком является ручная настройка сети WSN, что требует участия опытного специалиста. Выбор же открытого протокола WirelessHART позволяет реализовывать полностью самоорганизующиеся и самовосстанавливающиеся сети. Существенным при выборе этого протокола является то, что все функции безопасности программного слоя приемо-передачи данных реализуются поставщиком в обязательном порядке и, следовательно, на системном уровне необходимый уровень отказобезопасности коммуникационной среды выполняется также в обязательном порядке.

Протокол NB-Fi национального стандарта РФ беспроводной передачи данных на основе узкополосной модуляции радиосигнала обеспечивает отечественным разработчикам сенсорных сетей конкурентоспособность внедрения отечественных сенсорных сетей благодаря заявляемой низкой стоимости радиопередатчиков для устройств и возможность их производства в России. Протокол NB-Fi, относящийся к технологии LPWAN (энергоэффективная сеть дальнего радиуса действия), позволяет реализовывать сеть с устойчивой передачей данных на расстояния до 10 км в условиях присутствия препятствий и до 30 км в случае их отсутствия. Однако на настоящий момент неизвестна сертификация его отказобезопасности.

Протокол WirelessHART использует четыре верхние уровня сетевой модели OSI. Он обладает существенными преимуществами по сравнению с другими протоколами радиосвязи ограниченной мощности, обеспечивая надежную эксплуатацию приемопередающих каналов связи.

Важным достоинством его применения является то, что существующие проводные HART-приборы можно оснастить беспроводными модулями передачи данных, позволяющими интегрировать проводные HART-устройства в беспроводную сеть. Тем самым обеспечивается единый доступ к проводным и беспроводным приборам через беспроводный шлюз сбора данных. Благодаря использованию единого прикладного уровня в модели OSI (ISA100) все поддерживающие его HART-приборы оказываются взаимно совместимыми, что позволяет выполнять по единому алгоритму калибровку, настройку и диагностику приборов. Это особенно важно, так как миллионы проводных средств автоматизации по всему миру работают с использованием протокола HART.
Преимуществом стандарта ISA100.11а является то, что устанавливаемые временные интервалы связи имеют возможность on-line конфигурирования. Это позволяет поддерживать три основных режима работы каналов связи: скачкообразное изменение частоты между каналами, медленное изменение частоты от канала к каналу и гибридная перенастройка частоты. Для расширения возможности перенастройки частоты с использованием методов DSSS и FHSS находит применение технология CCA (Clear Channel Assessment), короткого времени передачи и функции занесения в «черный список» [18]. Благодаря такой гибкости в сенсорной сети могут обслуживаться одновременно как приборы, которые передают данные периодически или спорадически с основным временем в выключенном состоянии, так и приборы, которые требуют синхронизации по времени. Такой режим работы не требует синхронизации сетевых узлов по времени и позволяет обеспечивать высокую надежность коммуникации без дополнительных затрат энергии батареи [19].

Применение WSN в системах противоаварийной защиты НГО существенно зависит от отказобезопасности коммуникационной среды. Несмотря на то, что функциональная отказобезопасность программного слоя подготовки и передачи данных сертифицируется за счет применения стандартного профиля PROFIsafe, который осуществляет контроль времени, контроль нумерации кодовых посылок, контроль подписи сообщений и дополнительный контроль целостности данных, для обеспечения заявляемой SIL этого недостаточно. Электромагнитные помехи и физические препятствия распространения радиоволн ограничивают применимость WSN в СПАЗ. Дополнительным ограничением СПАЗ на безлюдных объектах НГО является существенное время недоступности (DTU) обслуживания (например, при замене батареи или техническом обслуживании отдельного модуля) из-за удаленности и труднодоступности НГО. Большинство предлагаемых технических решений на основе применения автономных сенсоров базируется на использовании не лицензируемых радиочастот 2,4 ГГц/434 МГц/868 МГц. Основное различие между данными ISM-диапазонами определяется частотой излучения и, как следствие, свойствами радиоволн. Наиболее «спокойным» относительно помех на текущий момент представляется диапазон 868 МГц, потому что безлицензионным в РФ он стал относительно недавно по решению ГКРЧ № 18-46-03-1 от 11 сентября 2018 г. [20]. Этот диапазон частот пока «не загружен» применением. Кроме того, по сравнению с СВЧ диапазоном более длинные волны (868 МГц) имеют меньшую интенсивность затухания и тем самым обеспечивается большая дальность передачи с увеличением «проницаемости» сигналов сквозь преграды. Поэтому применительно к задаче сбора данных средствами беспроводного управления и контроля наиболее оптимальным решением в настоящее время является использование диапазона 868 МГц.

Однако, несмотря на технические преимущества при передаче данных с частотой радиоволн 868 МГц, 
многие производители промышленных сетей с радиочастотной телекоммуникацией применяют в своих решениях частоту 2,4 ГГц, которая позволяет использовать более компактные и менее энергоемкие передатчики [21].

Низкотемпературное исполнение устройств сенсорных сетей в линейке сенсорных устройств компании Emerson позволяет использовать беспроводные приборы без дополнительного обогрева. В других исполнениях для повышения окружающей температуры сенсорного узла применяются специальные необогреваемые чехлы. Однако в условиях крайнего севера (при температурах ниже $-40{ }^{\circ} \mathrm{C}$ ) применение автономных сенсоров ограничивается условиями применимости электроники радиопередающих модулей датчиков. Самые низкие температурные показатели имеет оборудование КИПиА в линейке компании Emerson $\left(-60{ }^{\circ} \mathrm{C}\right.$ для датчика давления Rosemount $3051 \mathrm{~S},-55{ }^{\circ} \mathrm{C}$ для датчика температуры Rosemount $648 ;-40{ }^{\circ} \mathrm{C}$ для датчика уровня Rosemount 3308), что позволяет использовать беспроводные приборы без дополнительного обогрева в районах с суровым климатом. Поэтому их применение оправдано для районов, климатически неблагоприятных для применения автономных сенсоров

Согласно ГОСТ Р ИСО/МЭК 29182-1-2018, сенсорные сети могут иметь статическую топологию или динамически реконфигурироваться путем добавления или удаления сенсорных узлов. Таким образом, может поддерживаться динамическая топология сенсорной сети. Самоорганизация и самовосстановление являются атрибутами беспроводных сенсорных сетей и тесно связаны с динамической сетевой топологией. Самоорганизация сенсорной сети - это ее способность формировать новый сетевой граф телекоммуникации без необходимости вмешательства человека. Сетевой граф устанавливает, с какими сенсорными узлами связывается данный сенсорный узел. С другой стороны, самовосстановление - это способность сети восстанавливаться после сбоев сенсорных узлов или каналов связи. После того, как сеть самоорганизуется или восстановится, в ней могут быть налажены необходимые связи, и сенсорная сеть может выполнять свою функцию на приемлемом уровне [22]. У компании Yokogawa самоорганизация сенсорной сети обеспечена на уровне связи устройств сбора данных $[15,16]$.

Такие компании, как Emerson, Endress+Hauser, Siemens, для самоорганизации сети используют в своих решениях динамическую топологию (Mesh) [11]. Эта способность позволяет любому узлу сети выполнять функции передачи пакетов данных с другим участникам сети путем их ремаршрутизации, что в ряде применений улучшает стабильность передачи данных. Динамическая топология сети значительно упрощает процесс планирования сети, повышает надежность сети, т. к. каждый узел сети может выполнять функции ретранслятора и таким образом может служить коммутатором для соседних узлов. Такая топология сети обеспечивает надежную передачу данных, отлично масштабируется и обеспечивает са- морезервирование, поскольку возможно различное перенаправление данных от одного узла к точке назначения, минуя неисправные (недостигаемые) узлы. Однако, в сравнении со статической топологией, для ее реализации требуется заметно больше энергетических затрат, что значительно уменьшает срок службы элементов питания. Поэтому на практике такое решение ограничено в применении.

Самой распространенной сетевой топологией соединения сенсоров, предлагаемых ведущими компаниями в настоящее время, является «звезда» с одним центральным или маршрутизирующим узлом. Все конечные узлы такой звезды выполняют только одну функцию - передачу данных центральному узлу. С использованием топологии «звезда» отказ какоголибо конечного устройства не ведет к отказу сети, так как каждое конечное устройство связано только с центральным узлом. Достоинством такой топологии является меньшее потребление энергии, так как после отправки данных в соответствии с установленными алгоритмами передачи данных они возвращаются в спящий режим.

При использовании сенсорных сетей в АСУТП необходимо учитывать временные ограничения работы полевого оборудования, так как датчики осуществляют измерение и передачу значений с дискретизацией от 1 (0,5 сек у Honeywell) до 60 сек. Во время, отличное от измерения и передачи данных, для снижения энергопотребления прибор автоматически переводится в режим «Сон». Такая особенность технологии автономных сенсоров накладывает ограничения в сферах применения в АСУТП нефтегазодобычи, в частности, в системах противоаварийной защиты (СПАЗ), на узлах коммерческого учета быстроменяющихся технологических процессов, в замкнутых контурах управления.

При проектном выборе автономных сенсоров необходимо учитывать особенности рельефа (особенно если автоматизация охватывает удаленные друг от друга НГО), так как радиусы устойчивой работы устройств связи и оборудования могут значительно снизить область охвата автоматизацией объектов управления. В настоящее время существует проблема с прохождением сигнала через листву деревьев, так как падает дальность передачи данных. Можно ожидать, что совершенствование антенн может решить эту проблему. Так, компанией Emerson предлагается применение удлиненных антенн, что позволяет значительно расширить область охвата сенсорной сетью района нефтегазодобычи. Использование комплексов коммуникационного оборудования (до 5 ретрансляторов) также позволяет достигать расширения сети до 25 км. Компания Honeywell предлагает решения по использованию всенаправленных и остронаправленных внешних антенн. Возможна совместимость с производителями коммуникационного оборудования других фирм с поддержкой протокола ISA100.11a, что делает применение решений производителя более гибкими [23, 24].

Однако нельзя быть полностью уверенным в том, что радио канальные устройства могут быть доста- 
точно надежными и безопасными: распространение сигнала радиоволн может быть нарушено как природными, так техногенными причинами. Известна практика пилотных проектов беспроводных сенсоров на объектах добычи углеводородов компаний ОАО «Томскгазпром», ОАО «Лукойл-Коми», АО Самаранефтегаз, АО «Транснефть» и др. Так, в проектах БСС ОАО «Томскгазпром» в 2012 г. для автоматизации новых газовых и нефтяных скважин использовались:

- автономные датчики давления 3051S для измерения линейного давления, затрубного давления, буферного давления нефтяных и газовых скважин;

- автономные датчики температуры 648 для измерения температуры после штуцера газовых скважин;

- шлюз 1420 для сбора данных сенсорных датчиков. Датчики, оборудованные термочехлами без обогрева, были установлены непосредственно на оборудовании устья скважины через вентильные блоки или защитные гильзы. WSN оборудование было установлено на месторождениях Мыльджинское, СевероВасюганское, Болтное, Казанское, СевероОстанинское. Минимальная температура за две зимы эксплуатации составила $-43^{\circ} \mathrm{C}$.

Согласно отзывам ОАО «Томскгазпром», беспроводные датчики зарекомендовали себя как высоконадежные средства автоматизации. Сбоев не наблюдалось, влияния температурных пиков замечено не было. Отмечается легкость и удобство монтажа и эксплуатации беспроводных датчиков.

Проанализировав практики применения беспроводных сенсоров, можно сделать вывод, что применение WSN на объектах нефте- и газодобычи перспективно.

Однако при прямом сравнении капитальных вложений ни одна из рассматриваемых беспроводных измерительных систем не показала достаточной экономической эффективности по сравнению с проводным аналогом для достижения окупаемости в рамках прямой замены проводной системы на беспроводную. Следует иметь в виду, что эстакады на месторождениях устанавливаются не только для кабельных проводок передачи данных. Дополнительные способы достижения экономии на капитальных затратах WSN выявлены не были, однако существует возможность экономии на операционных затратах, в частности, связанных с ремонтом скважин.

Таким образом, в настоящее время беспроводные измерительные системы пока не могут быть рекомендованы для замены проводных аналогов. Нет экономической целесообразности реконструкции существующих объектов с их использованием в рамках проектов, в которых возникает необходимость вос-

\section{СПИСОК ЛИТЕРАТУРЬ}

1. Mutiu Adesina Adegboye, Wai-Keung Fung, Aditya Karnik Recent advances in pipeline monitoring and oil leakage detection technologies: principles and approaches // Sensors. - 2019. V. 19. - № 2548 - P. 1-36. становления ветхих кабельных эстакад либо строительство новых эстакад.

\section{Заключение}

Выполненный анализ перспектив применения автономных сенсоров и сетей на их основе показал, что основными преимуществами применения автоматизации в нефтегазовой отрасли на основе автономных сенсоров являются:

- отсутствие необходимости в прокладке кабельных каналов передачи данных;

- отсутствие необходимости выполнения комплексного инструментального контроля для установки/модернизации кабельных эстакад; в некоторых случаях это ведет к отказу в использовании затратных эстакадных конструкций кабельных проводок, что снижает стоимость работ по автоматизации протяженных объектов управления;

- значительное снижение объема инжиниринговых работ при обследовании объектов управления;

- снижение времени на проектирование систем.

Развертывание беспроводной сенсорной сети требует меньших трудовых и временных затрат, что может стать важным фактором в труднодоступных местах добычи углеводородов.

Однако на данный момент их применение ограничено из-за установленных отраслевых нормативов в области АСУТП для опасных производственных объектов нефтегазодобычи. Это, в частности, связано с проблемами отказобезопасности беспроводной среды передачи данных, значительной задержкой технического обслуживания в случае удаленности и труднодоступности технологических объектов управления.

В результате выполненного исследования хотя и не удалось выявить значимую экономическую эффективность замены проводных каналов связи на их беспроводной аналог, тем не менее можно ожидать, что в результате совершенствования беспроводных сенсоров их применение окажется экономически выгодным.

В настоящее время использование автономных беспроводных сенсоров будет целесообразным, если:

- существует необходимость быстрого монтажа или демонтажа контрольного оборудования;

- есть потребность в использовании мобильного измерительного комплекса (например, для мониторинга температуры и давления при пропарке резервуаров или давления и загазованности при работе на магистральных трубопроводах);

- существует необходимость оперативного увеличения количества точек измерения в сжатые сроки;

- есть необходимость скорейшего восстановления работы технологического процесса (например, после пожара, после аварийной ситуации).

2. Развитие перспективной автоматизации в нефтегазовой отрасли / Е.И. Громаков, Т.Е. Мамонова, А.В. Лиепиньш, А.Н. Рымшин // Нефтяное хозяйство. - 2019. - № 10. - С. 98-102.

3. Мамонова Т.Е. Определение кратковременных утечек в нефтепродуктопроводах // Нефтяное хозяйство. - 2014. № 4. - С. 96-99. 
4. Мамонова Т.Е. Гидродинамические модели в задачах определения утечек из нефтепродуктопроводов // Нефтяное хозяйство. - 2015. - № 4. - С. 92-95.

5. Mamonova T.E., Vasilyeva T.N. Artificial intelligence in problems of leak definition from the oil pipeline // Proceedings of 2014 International Conference on Mechanical Engineering, Automation and Control Systems, MEACS 2014. - P. 6986846

6. Mamonova T.E. oil product loss identification per pressure variations in time at four controlled pipe cross-sections // Automation and Remote Control. - 2020. - V. 81. - № 1. P. 192-198.

7. A three-dimensional finite element analysis of the temperature field during laser melting of metal powders in additive layer manufacturing / I.A. Roberts, C.J. Wang, R. Esterlein, M Standford, D. Manors // International Journal of Machine Tools and Manu-facture. - 2009. - V. 49. - № 12. - P. 16-23.

8. Research on evaluation method of wellbore hydrate blocking degree during deepwater gas well testing / Wenyuan Liua Jinqiu Hu, Xiangfang Li, Fengrui Sun, Zheng Sun, Yunjian Zhou // Journal of Natural Gas Science and Engineering. - 2018. № 59. - P. 168-182.

9. Pipeline leakage localization based on distributed FBG hoop strain measurements and support vector machine / Z. Jia, Z. Wang, W. Sun, Z. Li // Optik. - 2019. - № 176. - P. 1-13.

10. Bernstein J., Macias D. Engineering new-product success. The new-product pricing process at Emerson. The new-product pricing process is the foundation for a set of key marketing activities // Industrial Marketing Management. - 2002. - № 31. - P. 51-64.

11. A novel MAC proposal for critical and emergency communications in Industrial Wireless Sensor Networks / Mohsin Raza, Hoa Le-minh, Nauman Aslam, Sajjad Hussain // Computers and Electrical Engineering. - 2018. - № 72. - P. 976-989.

12. Application of wireless technology for control: a WirelessHART perspective / Sabo Miya Hassan, Rosdiazli Ibrahim, Kishore Bingi, Tran Duc Chung, Nordin Saad // 2016 IEEE International Symposium on Robotics and Intelligent Sensors, IRIS 2016. Tokyo, Japan, 17-20 December 2016, Procedia Computer Science. -2017 - №. 105 . - P. 240 - 247.

13. Accutech. Автономные беспроводные измерительные устройства / Shneider Electric. 2013. - 12 c. URL: https://trss.pro/ catalog/Accutech/doc/Accutech_brochure.pdf (дата обращения: 22.06.2021).

14. Machine learning for wireless communications in the Internet of Things: A comprehensive survey / J. Jagannath, N. Polossky,
A. Jagannath, F. Restuccia, T. Melodia // Ad Hoc Networks. 2019. - № 93. - P. 101913.

15. Беспроводные контрольно-измерительные приборы / Yokogawa Electric Corporation. Field Instruments BU, December, 2010. 34 c. URL: http://yoko.kip-postavka.ru/GS/ Wireless ISA100ru.pdf (дата обращения: 22.06.2021).

16. Siemens. Increasing transparency and flexibility at the field level // Siemens AG, 2012. - 14 p. URL: https://www.siemenspro.ru/docs/kip/common/Broshures/E20001-A960-P710-X7600.pdf (дата обращения: 26.04.2021).

17. Energy consumption analysis of WirelessHART adaptor for industrial wireless sensor actuator network / Tran Duc Chung, Rosdiazli Ibrahim, Vijanth Sagayan Asirvadam, Nordin Saad, Sado Miya Hassan // 2016 IEEE International Symposium on Robotics and Intelligent Sensors, IRIS 2016. - Tokyo, Japan, 17-20 December 2016, Procedia Computer Science. - 2017 № 105. - P. 227-234.

18. Industrial wireless automation: overview and evolution of WIAPA / Y. das Neves Validao, G. Kunzel, I. Muller, C.E. Pereira // IFAC PapersOnLine. - 2018. - V. 51. - № 10. - P. 175-180.

19. Rehman K., Nawaz F. Remote pipeline monitoring using Wireless Sensor Networks // Proc. of the International Conference on Communication, Computing and Digital Systems (C-CODE). Islandbad, Pakistan, 8-9 March 2017; IEEE: Piscataway, NJ, USA, 2017. - P. 32-37.

20. Магические 2.4 ГГц., 2011. URL: http://wmusers.ru/2011/06/29/ magicheskie-2-4-ggc/ (дата обращения: 22.06.2021).

21. Датчик-газоанализатор стационарный ДГС ЭРИС-210 Модификация 1 Руководство по эксплуатации, 2018. - 42 с. URL: https://eris.nt-rt.ru/images/manuals/dgs210.pdf (дата обращения: 26.06.2021)

22. Каталог оборудования компании Yokogawa, 2016. - 27 c. URL: http://www.yokogawa.ru/products/kip/wireless_solutions/ (дата обращения: 26.06.2021).

23. Бражук А. Построение беспроводных локальных сетей на основе ячеистой топологии // Технологии и стандарты. Беспроводные технологии. - 2006. - № 4. - C 24-25. URL: https://wireless-e.ru/wireless-networks/mesh/ (дата обращения: 26.06.2021).

24. Leitao P., Colombo A.W., Karnouskos S. Industrial automation based on cyber-physical systems technologies: Prototype implementations and challenges // Computers in Industry. 2016. - V. 81. - P. 11-25.

Поступила 25.07.2021 2.

\section{Информация об авторах}

Мамонова T.E., кандидат технических наук, доцент отделения автоматизации и робототехники Национального исследовательского Томского политехнического университета.

Громаков Е.И., кандидат технических наук, доцент отделения автоматизации и робототехники Национального исследовательского Томского политехнического университета. 


\title{
UDC 608.2
}

\section{AUTONOMOUS SENSORS USING FOR AUTOMATION OF OIL AND GAS PRODUCTION}

\author{
Tatiana E. Mamonova ${ }^{1}$, \\ stepte@tpu.ru
}

\author{
Evgeniy I. Gromakov', \\ gromakov@tpu.ru \\ 1 National Research Tomsk Polytechnic University, \\ 30, Lenin avenue, Tomsk, 634050, Russia.
}

The relevance. The essential advantage of autonomous sensors when used to automate technological processes is the cabling absence. Suppliers believe that their implementation provides decline of capital costs for construction of projects (in particular, due to savings on cable products and materials), reduction of construction, installation and commissioning work time, energy consumption and operating costs reduction. The application prospect of design decisions automation using the radio and communication environment for data production had a promising potential for development. The introduction of network-centric technologies of automatic (automated) control on the basis of intelligent sensor networks, first of all, will allow increasing the efficiency of hydrocarbon raw materials extraction in the short term in hard-to-reach territories, will reduce the need for mandatory man presence at technological sites. All this, in its turn, can significantly change the design decisions for automation of oil and gas production objects.

The main aim of the research is identification of the main obstacles when using autonomous sensors for automation of oil and gas production, comparative analysis of the main manufacturers of autonomous sensors.

Objects: the network-centric technologies of automatic (automated) control based on intelligent sensor networks for oil and gas production. Methods: system analysis of domestic and foreign markets of autonomous sensors and networks.

Results. The authors have analyzed the use of autonomous sensors and networks based on them and identified the main advantages of using automation in the oil and gas industry based on autonomous sensors. The paper demonstrated the conditions under which the use of autonomous wireless sensors at oil and gas facilities is promising. As a result of the study, it was not possible to identify significant economic efficiency of automation of typical oil and gas production processes by replacing all wired automation with its wireless analogue. Currently, the use of autonomous sensor networks in the field of automatic process control systems for hazardous oil and gas production facilities is also limited, as there are difficulties in certifying functional safety. This, in particular, is due to the problems with the failure safety of the radio communication environment, as well as a significant delay in maintenance in case of remote and inaccessible technological control facilities.

\section{Key words:}

WSN, wireless sensor, smart fields, distributed control system, instrumentation

\section{REFERENCES}

1. Mutiu Adesina Adegboye, Wai-Keung Fung and Aditya Karnik. Recent advances in pipeline monitoring and oil leakage detection technologies: principles and approaches. Sensors, 2019, vol. 19, no. 2548, pp. 1-36.

2. Gromakov E.I., Mamonova T.E., Liepinsh A.V., Rymshin A.N Development of perspective automation in the oil and gas industry. Bulletin of the Oil Industry, 2019, no. 10, pp. 98-102. In Rus.

3. Mamonova T.E. Definition of short-term leakage in product pipelines. Bulletin of the Oil Industry, 2014, no. 4, pp. 96-99. In Rus.

4. Mamonova T.E. Using the hydrodynamic models in problems of leaks definition from oil pipelines. Bulletin of the Oil Industry, 2015, no. 4, pp. 92-95. In Rus.

5. Mamonova T.E., Vasilyeva T.N. Artificial intelligence in problems of leak definition from the oil pipeline. Proceedings of 2014 International Conference on Mechanical Engineering, Automation and Control Systems, MEACS 2014. pp. 6986846.

6. Mamonova T.E. oil product loss identification per pressure variations in time at four controlled pipe cross-sections. Automation and Remote Control, 2020, vol. 81, no. 1, pp. 192-198.

7. Roberts I.A., Wang C.J., Esterlein R., Standford M., Manors D. A three-dimensional finite element analysis of the temperature field during laser melting of metal powders in additive layer manufacturing. International Journal of Machine Tools and Manufacture, 2009, vol. 49, no. 12, pp. 16-23.

8. Wenyuan Liua, Jinqiu Hu, Xiangfang Li, Fengrui Sun, Zheng Sun, Yunjian Zhou. Research on evaluation method of wellbore hydrate blocking degree during deepwater gas well testing. Journal of Natural Gas Science and Engineering, 2018, no. 59, pp. 168-182.

9. Jia Z., Wang Z., Sun, W., Li Z. Pipeline leakage localization based on distributed FBG hoop strain measurements and support vector machine. Optik, 2019, no. 176, pp. 1-13.
10. Bernstein J., Macias D. Engineering new-product success. The new-product pricing process at Emerson. The new-product pricing process is the foundation for a set of key marketing activities. Industrial Marketing Management, 2002, no. 31, pp. 51-64.

11. Mohsin Raza, Hoa Le-minh, Nauman Aslam, Sajjad Hussain. A novel MAC proposal for critical and emergency communications in Industrial Wireless Sensor Networks. Computers and Electrical Engineering, 2018, no. 72, pp. 976-989.

12. Sabo Miya Hassan, Rosdiazli Ibrahim, Kishore Bingi, Tran Duc Chung, Nordin Saad. Application of wireless technology for control: a WirelessHART perspective. 2016 IEEE International Symposium on Robotics and Intelligent Sensors, IRIS 2016. Tokyo, Japan, 17-20 December 2016, Procedia Computer Science, 2017, no. 105. pp. 240-247.

13. Accutech. Avtonomnye besprovodnye izmeritelnye ustroystva [Accutech. Self-contained wireless measuring devices]. Available at: https://trss.pro/catalog/Accutech/doc/Accutech_brochure.pdf (accessed 22 July 2021).

14. Jagannath J., Polossky N., Jagannath A., Restuccia F., Melodia T. Machine learning for wireless communications in the Internet of Things: a comprehensive survey. Ad Hoc Networks, 2019, no. 93. pp. 101913.

15. Besprovodnye kontrolno-izmeritelnye pribory [Wireless instrumentation]. Yokogawa Electric Corporation. Field Instruments $B U$. December, 2010. 34 p. Available at: http://yoko.kippostavka.ru/GS/Wireless_ISA100ru.pdf (accessed 22 July 2021).

16. Siemens. Increasing transparency and flexibility at the field level. Siemens $A G, 2012$. 14 p. Available at: https://www.siemenspro.ru/docs/kip/common/Broshures/E20001-A960-P710-X7600.pdf (accessed 22 July 2021).

17. Tran Duc Chung, Rosdiazli Ibrahim, Vijanth Sagayan Asirvadam, Nordin Saad, Sado Miya Hassan. Energy consumption analysis of WirelessHART adaptor for industrial wireless sensor actuator 
network. 2016 IEEE International Symposium on Robotics and Intelligent Sensors, IRIS 2016. Tokyo, Japan, 17-20 December 2016 Procedia Computer Science, 2017, no. 105. pp. 227-234.

18. Das Neves Validao Y., Kunzel G., Muller I., Pereira C.E. Industrial wireless automation: overview and evolution of WIA-PA. IFAC PapersOnLine, 2018, vol. 51, no. 10. pp. 175-180.

19. Rehman K., Nawaz F. Remote pipeline monitoring using Wireless Sensor Networks. Proceedings of the International Conference on Communication, Computing and Digital Systems (C-CODE). Islandbad, Pakistan, 8-9 March 2017. IEEE: Piscataway, NJ, USA, 2017 . pp. 32-37.

20. Magicheskie 2.4 GGz 2011 [Magic $2.4 \mathrm{GHz}$. 2011]. Available at: http://wmusers.ru/2011/06/29/magicheskie-2-4-ggc/ (accessed 26 June 2021).

21. Datchik-gazoanalizator spetsialniy DGS ERIS-210. Modifikatsiya 1. Rukovodstvo po ekspluatatsii [Sensor-gas analyzer stationary DGS ERIS-210. Modification 1. Operation Manual]. 2018. 42 p. Avail-

\section{Information about the authors}

Tatiana E. Mamonova, Cand. Sc., associate professor, National Research Tomsk Polytechnic University. Evgeniy I. Gromakov, Cand. Sc., associate professor, National Research Tomsk Polytechnic University. able at: https://eris.nt-rt.ru/images/manuals/dgs210.pdf (accessed 26 June 2021)

22. Katalog oborudovaniya kompanii Yokogawa [Yokogawa Equipment Catalog]. 2016. 27 p. Available at: http://www.yokogawa. ru/products/kip/wireless_solutions/ (accessed 26 June 2021).

23. Braguk A. Postroenie besprovodnykh lokalnykh setey na osnove yacheistoy topologii [Building wireless LANs based on mesh topology]. Tekhnologii i standarty. Besprovodnye tekhnologii, 2006, no 4, pp. 24-25. Available at: https://wireless-e.ru/wirelessnetworks/mesh/ (accessed 26 June 2021).

24. Leitao P., Colombo A.W., Karnouskos S. Industrial automation based on cyber-physical systems technologies: prototype implementations and challenges. Computers in Industry, 2016, vol. 81, pp. 11-25.

Received: 25 July 2021. 